NOUVELle

\section{La mécanique de la division cellulaire ou comment scinder une sphère en deux?}

Jean-Yves Tinevez ${ }^{1}$, Guillaume Salbreux ${ }^{2}$, Ewa Paluch ${ }^{3}$
> La division cellulaire est un événement clé de la vie d'une cellule et suscite la curiosité des scientifiques depuis plus d'un siècle [1]. Elle est le résultat d'une chorégraphie cellulaire bien ordonnée impliquant la formation du fuseau mitotique, la séparation des chromosomes et des autres composants cellulaires, suivies de la cytocinèse, qui est la division physique de la cellule mère en deux cellules filles. Même s'ils sont contrôlés par les voies de signalisation qui régissent la progression du cycle cellulaire, les changements de forme cellulaire au cours de la cytocinèse sont avant tout un processus mécanique [2]. La majorité des cellules animales s'arrondissent au moment de l'entrée en mitose et présentent une forme essentiellement sphérique en métaphase [3]. La séparation des chromosomes en anaphase est associée à un progressif allongement de la forme de la cellule. La cellule forme ensuite un anneau d'actine-myosine à l'équateur, qui se contracte, donnant à la cellule une forme en 8 , avant qu'elle ne se scinde en deux.

\section{Mécanique de la cytocinèse}

La structure responsable de ces changements de forme est le cortex cellulaire. Le cortex est un réseau réticulé de filaments d'actine qui forment une couche mince directement sous la membrane plasmique de la cellule. Le cortex contient une forte concentration de moteurs de type myosine II, qui en font une structure très contractile. Des modifications locales de la densité ou de la contractilité corticale sont à la base de nombreux types de déformations cellulaires $[4,5]$.
Les changements de forme caractéristiques de la division cellulaire sont le résultat de modifications contrôlées des propriétés mécaniques du cortex d'actine-myosine. À l'entrée en mitose, la contractilité corticale augmente, entraînant l'arrondissement de la cellule. En anaphase, le cortex s'accumule progressivement à l'équateur de la cellule, alors que la densité et la contractilité du cortex diminuent aux pôles. Alors qu'elles s'accumulent à l'équateur, l'actine et la myosine se réorganisent en un anneau contractile formé de filaments d'actine tendant à s'aligner (Figure IA). L'anneau contractile a alors une certaine ressemblance avec les sarcomères des muscles. Sa contraction est responsable de la division de la cellule en deux. La plupart des travaux étudiant la mécanique de la cytocinèse se sont focalisés sur la formation et l'ingression de l'anneau équatorial [6]. L'anneau est en effet le principal élément produisant des forces pendant la cytocinèse. Cependant, un cortex d'actomyosine demeure présent aux pôles de la cellule tout au long de la cytocinèse. La contribution de ce cortex polaire à la mécanique de la division a été peu étudiée. Des travaux dans l'amibe Dictyostelium discoideum indiquent qu'il oppose une résistance physique à l'ingression de I'anneau de division [7]. Cependant, un rôle actif du cortex polaire dans la mécanique de la division n'a pas été envisagé. Dans un travail récent, nous nous sommes intéressés à la contribution des forces contractiles exercées par le cortex polaire à la mécanique de la division cellulaire [8].
${ }^{1}$ Institut Pasteur, Imagopole, plate-forme d'imagerie dynamique,

25-28, rue du docteur Roux, 75015 Paris, France ;

${ }^{2}$ Max Planck Institute for the Physics of Complex Systems, Nöthnitzer St 38, 01187 Dresden, Allemagne ;

${ }^{3}$ Max Planck Institute of Molecular Cell Biology and Genetics, Pfotenhauerstrasse 108, 01307 Dresden, Allemagne et International Institute of Molecular Cell Biology, Warsaw, Pologne.

tinevez@pasteur.fr

\section{Tension de surface}

et instabilités de forme

Le cortex génère une force analogue à une tension de surface : chaque élément de cortex est un élément contractile qui crée une force tangentielle à la surface de la cellule. La résultante des forces créées par tout le cortex tend à diminuer la surface totale, ce qui peut se comprendre par une analogie grossière avec un ballon de latex. Une fois gonflé et libre de toute force extérieure, le ballon prendra une forme sphérique. Le latex de sa membrane est étiré, et comme pour un élastique, il tend à se contracter. Ce faisant, il presse sur le volume d'air dans le ballon, et génère une pression interne. Cette pression est reliée à la tension $T$ résultant de l'étirement du ballon par la loi de Laplace: $P=2 T / R$, où $R$ est le rayon du ballon. Pour maintenant simuler la division cellulaire, on peut passer un fil autour de l'équateur du ballon. En resserrant le fil, on espère créer un sillon, qui partagera le volume en deux, mimant ainsi l'activité de l'anneau contractile des cellules en division. Mais pour peu qu'il existe la moindre différence de taille entre les deux lobes du ballon, la loi de Laplace entraîne un gradient de pression entre les deux lobes, créant un flux d'air du plus petit lobe vers le plus grand. Si cet effet domine sur l'élasticité du ballon, le petit lobe se déverse alors totalement dans le plus gros, le ramenant à la forme sphérique initiale (Figure 1B). 


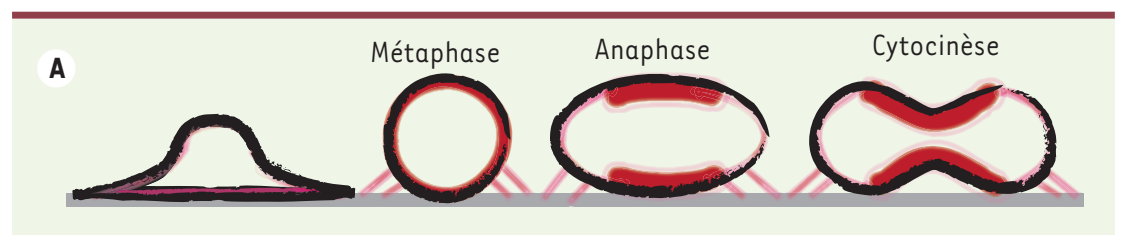

B

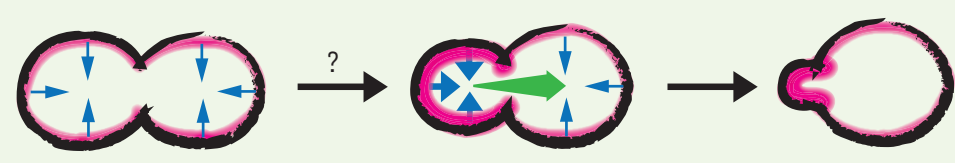

c
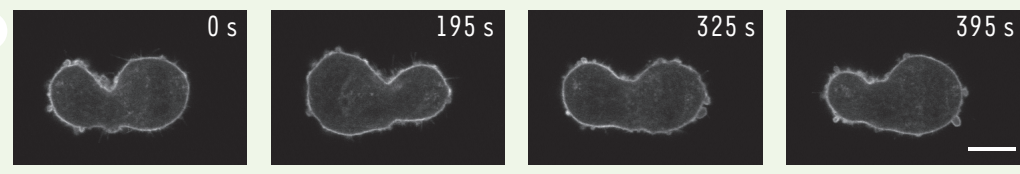

D
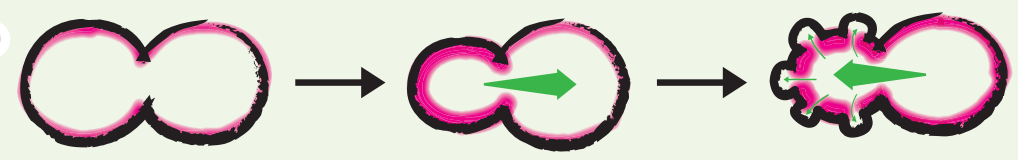

Figure 1. Contrôle de la forme cellulaire pendant la division cellulaire. A. Vision schématique de la cellule en division. Le cortex d'actomyosine est responsable de l'arrondissement de la cellule en métaphase. En anaphase, il est recruté à l'équateur, formant un anneau contractile et donne à la cellule sa forme en 8 . À ce stade, un cortex d'actomyosine est toujours présent aux pôles de la cellule. B. Exemple d'instabilité générée par la tension de Laplace : tant que les deux lobes ont la même taille, la pression (donnée par $P=T / 2 R$ où $R$ est le rayon du lobe) de chaque lobe s'équilibre. Dès qu'un des lobes devient plus petit que l'autre, il génère une pression plus forte, qui le conduit à se contracter et à diminuer encore son rayon. Au final, le petit lobe se déverse totalement dans le grand. $C$. Observation des instabilités de forme dans une cellule en division où la tension corticale aux pôles a été augmentée par déplétion de l'anilline (cellule fibroblastique de souris L929, filaments d'actine marqués par LifeAct-GFP, barre d'échelle : $10 \mu \mathrm{m}$ ). Chaque lobe se contracte alternativement, générant un flux cytosolique d'un lobe à l'autre. On observe la présence de blebs sous la forme de petites protrusions sphériques. Images : Andrea Pereira. D. Les blebs contribuent à stabiliser la forme de la cellule en division: Iorsqu'un lobe se contracte, l'enrichissement local en cortex induit sa rupture et/ou son détachement de la membrane. La pression intracellulaire appuie alors sur la membrane libre et génère des blebs, de petites protrusions membranaires initialement dépourvues de cortex. La naissance de ces protrusions permet de relâcher l'excédent de pression intracellulaire et inverse le flux cytosolique. Ceci limite l'amplitude de la contraction et empêche l'instabilité de se développer.

La division cellulaire a des spécificités qui limitent grandement la portée de cette analogie. En particulier, la cellule contient dans son volume un certain nombre de composants élastiques (faisceau mitotique, réseau de filaments intermédiaires, etc.) qui vont s'opposer à la contractilité corticale et limiter l'instabilité décrite par notre des cellules en division. Ces oscillations restent suffisamment modestes pour ne pas empêcher les cellules de poursuivre la division cellulaire. Dans un petit nombre de cas toutefois, leur amplitude augmente jusqu'à ce que I'anneau de constriction soit rejeté à l'un des pôles, résultant en un échec de la cytocinèse. Il est possible d'augmenter ce pourcentage en altérant la tension corticale des deux pôles (Figure IC), ou d'un seul des pôles, soit en y déposant localement la cytochalasine $D$ qui dépolymérise les filaments d'actine, soit par ablation directe d'un point du cortex en utilisant un laser pulsé. Le déséquilibre de tension qui en résulte induit des oscillations catastrophiques qui empêchent la division cellulaire, résultant en la génération de cellules binucléées. En combinant une analyse quantitative des films des oscillations avec un modèle physique, nous avons pu montrer que ces oscillations sont déclenchées par une instabilité comparable à celle décrite plus haut pour le ballon, et sont entretenues par l'effet combiné de la tension corticale, de l'élasticité de la cellule et de la dynamique d'assemblage du cortex d'actine.

\section{Les blebs : des valves de pression?}

La cellule en division doit donc se prémunir contre ces instabilités provenant de la contractilité du cortex d'actomyosine. Un bon moyen de protection semble être l'apparition de blebs. Les blebs sont de petites protrusions sphériques apparaissant au niveau du cortex, initialement constituées de membrane nue. Celle-ci se garnit en quelques secondes d'un nouveau cortex d'actomyosine, conduisant à la rétraction du bleb. La formation de blebs a longtemps été associée à l'apoptose, où ils jouent un rôle important dans la fragmentation du matériel nucléaire [9]. Cependant, ils sont aussi communément observés pendant la division cellulaire [10] ou pendant la migration, où ils peuvent remplacer le lamellipode à l'avant de la cellule [11]. 
Pour les cellules dont la forme oscille, on observe les blebs de manière alternée, d'un pôle à l'autre, suivant la contraction du petit lobe. Or la formation de blebs a pour effet de relâcher la tension du cortex et diminuer la pression qui en résulte [12]. II est donc plausible qu'ils contribuent par leur apparition à tempérer une augmentation de tension d'un lobe qui viendrait déstabiliser la cellule en division. Nous avons montré que si I'on bloque l'apparition des blebs en stabilisant le cortex d'actine de cellules contrôles par l'ajout de différentes lectines (wheat germ agglutinin et concanavaline A), la proportion de cellules qui oscillent et dont la division échoue augmente. De plus, l'induction de blebs dans des cellules oscillantes, par ablation au laser d'un pôle en train de se contracter, stoppe la contraction du pôle et inverse la direction de l'oscillation. Ces observations nous permettent de proposer un nouveau rôle pour les blebs lors de la division cellulaire : ils joueraient le rôle de valves de pression, relâchant la tension polaire dès que celle-ci dépasse un seuil risquant de déclencher des instabilités de forme (Figure 1D).

\section{Conclusion}

Lors de la cytocinèse, la cellule maintient une tension à ses pôles, peut-être pour conserver une forme sphérique et contrôler le volume des futures cellules filles. Cependant, les forces générées par la cellule pour maintenir sa forme peuvent aussi entraîner des instabilités mécaniques pouvant mener à l'échec de la division. II paraît naturel que l'évolution ait introduit des mécanismes de contrôle pour éviter à la cellule ce type d'erreur. \&n plus du rôle stabilisant joué par le fuseau mitotique qui contrôle la contractilité corticale lors de la division, il est possible que l'interaction entre la membrane et le cortex, à travers la formation de blebs capables de relâcher la tension du cortex, fournisse un mécanisme capable de stabiliser la forme cellulaire. Lorsque les divers mécanismes de stabilisation échouent, la cellule présente des instabilités de forme qui peuvent se manifester par des oscillations de forme. Notre modèle suggère que de telles oscillations sont un comportement dynamique intrinsèque d'un cortex contractile et dynamique agissant contre un milieu élastique. $\diamond$

The mechanics of the cellular division or how to split a sphere into two?

\section{CONFLIT D'INTÉRÊTS}

Les auteurs déclarent n'avoir aucun conflit d'intérêts concernant les données publiées dans cet article.

\section{RÉFÉRENCES}

1. Rappaport R. Cytokinesis in animal cells. Cambridge : Cambridge University Press, 1996.

2. Robinson DN, Spudich JA. Mechanics and regulation of cytokinesis. Curr Opin Cell Biol $2004 ; 16: 182-8$.

3. Stewart MP, Helenius J, Toyoda Y, et al. Opposing activities of hydrostatic pressure and the actomyosin cortex drive mitotic cell rounding. Nature $2011 ; 469$ : 226-30.

4. Bray D, White JG. Cortical flow in animal cells. Science $1988 ; 239: 883-8$

5. Mayer M, Depken M, Bois J, et al. Anisotropies in cortical tension reveal the physical basis of cortical flow in polarising C. elegans zygotes. Nature 2010; 467: 617-21.

6. Pollard TD. Mechanics of cytokinesis in eukaryotes. Curr Opin Cell Biol $2010 ; 22: 50-6$

7. Zhang W, Robinson DN. Balance of actively generated contractile and resistive forces controls cytokinesis dynamics. Proc Natl Acad Sci USA 2005; 102 : 7186-91.

8. Sedzinski J, Biro M, Oswald A, et al. Polar actomyosin contractility destabilises the position of the cytokinetic furrow. Nature $2011 ; 476$ : 462-6.

9. Croft DR, Coleman ML, Li S, et al. Actin-myosin-based contraction is responsible for apoptotic nuclear disintegration. J Cell Biol $2005 ; 168: 245-55$.

10. Strangeways T. Observations on the changes seen in living cells during growth and division. Proc $R$ Soc Lond Ser B 1922 ; 94 : 137-41.

11. Charras G, Paluch $\varepsilon$. Blebs lead the way: how to migrate without lamellipodia. Nat Rev Mol Cell Biol $2008 ; 9: 730-6$.

12. Tinevez JY, Schulze U, Salbreux G, et al. Role of cortical tension in bleb growth. Proc Natl Acad Sci USA 2009 ; 106 : 18581-6.

\section{NOUVELLE}

\section{Cellules épithéliales médullaires thymiques exprimant Aire}

\section{Acteurs clés dans l'induction de la tolérance des cellules T}

Magali Irla
Département de pathologie et immunologie, centre médical universitaire de Genève,

1 , rue Michel Servet, 1211 Genève, Suisse.

\section{Magali.Irla@unige.ch}

Nouvelle adresse : centre d'immunologie de Marseille Luminy, CNRS-Inserm-université de la Méditerranée, parc scientifique et technologique de Luminy, Case 906, 13288 Marseille Cedex 09, France.
> Le thymus, organe lymphoïde primaire, est le site majeur de la production des lymphocytes $T$ dont le rôle est essentiel dans la réponse immunitaire aux agents pathogènes et aux cellules tumorales. Cet organe coordonne les événements de sélection conduisant au développement d'un répertoire de lymphocytes $T$ fonctionnels non réactifs vis-à-vis du soi. Ces événements de sélection sont essentiels pour éviter la génération de cellules $T$ autoréactives, responsables du développement de maladies autoimmunes. Lors de leur différenciation dans le thymus, les cellules T dont le TCR ( $T$ cell receptor) réagit avec les antigènes
(Ag) du soi (cellules autoréactives) sont éliminées dans une région anatomique spécifique, appelée médulla. Les cellules épithéliales médullaires thymiques (mTEC) jouent un rôle clé dans l'élimination des cellules $T$ autoréactives grâce à leur propriété unique d'exprimer une grande diversité d’Ag spécifiques de 